\title{
APPLICATION OF ANSYS IN TEACHING FEM ON THE EXAMPLE OF A LOADED MARS ROVER CHASSIS
}

\author{
Małgorzata Łazuka', Ewa Łazuka² \\ 1 Students'Space Association, Faculty of Power and Aeronautical Engineering, Warsaw University of Technology, \\ Nowowiejska 24, 00-665 Warsaw, Poland, e-mail: lazuka.malgorzata@gmail.com \\ 2 Department of Applied Mathematics, Faculty of Fundamentals of Technology, Lublin University of Technology, \\ Nadbystrzycka 38, 20-618 Lublin, Poland, e-mail: e.lazuka@pollub.pl
}

Received: 2016.08.15

Accepted: 2016.10.26

Published: 2016.12.01

\begin{abstract}
The aim of the paper is to present the idea of using ANSYS software in teaching engineering students the finite element method. ANSYS is an extremely sophisticated engineering tool used in almost every engineering company nowadays. It is introduced to almost all engineering students during their studies. Using ANSYS knowingly requires perfect understanding of the finite element method that is responsible for ANSYS work. This paper presents how this professional software can help students master FEM. The first part of the paper introduces the finite element method. It also suggests a way of using ANSYS in teaching FEM on the example of a twodimensional restrained beam calculated in a simple computer program. The second part illustrates the effectiveness of ANSYS with sample FEM analysis of a planetary rover's chassis and presents basic fuctionalities of ANSYS Workbench Mechanical. Conducted calculations provide useful data about the model's suitability for its purpose and possibility of modifications.
\end{abstract}

Keywords: ANSYS, FEM, finite element method, numerical analysis, teaching, bending, beam, chassis

\section{INTRODUCTION}

Since our technical knowledge has developed and we have discovered and studied all the laws behind various phenomena, we started facing a growing problem: lack of sufficient computing power to simulate various complex processes with satisfactory accuracy. Therefore, we put a great deal of effort into two possible solutions to this problem: increasing the available computing power and reducing the computational complexity of considered issues. This way we led to a rapid and widespread development of numerical methods, which enable us to perform calculations and obtain approximate results significantly faster than exact solutions. Numerical methods occured essential in numerous engineering disciplines, i.a. mechanical and structural analysis, in which they are an inte- gral part of the finite element method (FEM), the most popular method of solving complex problems of mechanics $[2,6]$. One of the best and most popular tools for simulating materials' behaviour is ANSYS, which uses various complex numerical algorithms and FEM. It is an engineering analysis software system used i.a. for performing structural analysis, analysing fluid dynamics and designing electronics [1]. There is a great number of different ANSYS products, which makes the system present in career of almost every contemporary engineer. However, what also turns out to be surprisingly effective is teaching finite element method with the use of ANSYS.

The aim of this thesis is to demonstrate how powerful ANSYS is as an engineering software tool, as well as present the possibility of using ANSYS in teaching future engineers. 


\section{USING ANSYS IN TEACHING FEM}

FEM is a method of calculating some mechanical issues of deformable objects by diving them into small elements properly bound together, called finite elements [4]. This problem of model discretisation is one of the most difficult issues for students to understand in the finite element method. The impacts from the outside are usually known in advance and estimated with large safety factors. Determining the boundary conditions during FEM analysis in sophisticated programs like ANSYS is reduced to the knowledge of constraints imposed on the model without worrying about their mathematical form. However, choice of the type and number of finite elements has a great influence on the time needed to get the results and on their accuracy [6]. Therefore, choosing proper finite elements is a key issue during such calculations. It is crucial to choose a proper shape of the finite element and number of nodes on each of its edges [8]. The grid must be neither too dense, so that the calculations end in a reasonable time, nor too rare, so that the results do not differ too much from the exact solution. That is why the best strategy is to use rare grids in parts of the model where the considered vari- able (stress, magnetic field intensity etc.) will not change its value rapidly and to use dense grids in parts where we expect such changes to occur.

After introducing FEM and its main idea, principles and requirements theoretically during lectures, next step of teaching it is exploring the algorithm. At this point ANSYS may occur useful - however, only as a model to follow. Namely, an interesting idea is to conduct project classes, during which students will have the opportunity to create their own finite element method algorithm basing on their theoretical knowledge. The used model should not be complex - it ought to be just two-dimensional with simple boundary conditions and discretisation into few finite elements [3]. In case of structural analysis, an excellent example of such a model can be an ordinary, two-dimensional, restrained beam subjected to bending (presented in Figure 1).

Students shall be able not only to code the FEM algorithm step by step and optimise it, but also experiment with it by changing the problem's complexity. This exercise shall demonstrate how much our choice of the grid's density and type will affect the program running time and the accuracy of the results. In case of such a mini simulation program using FEM, of course the nu-

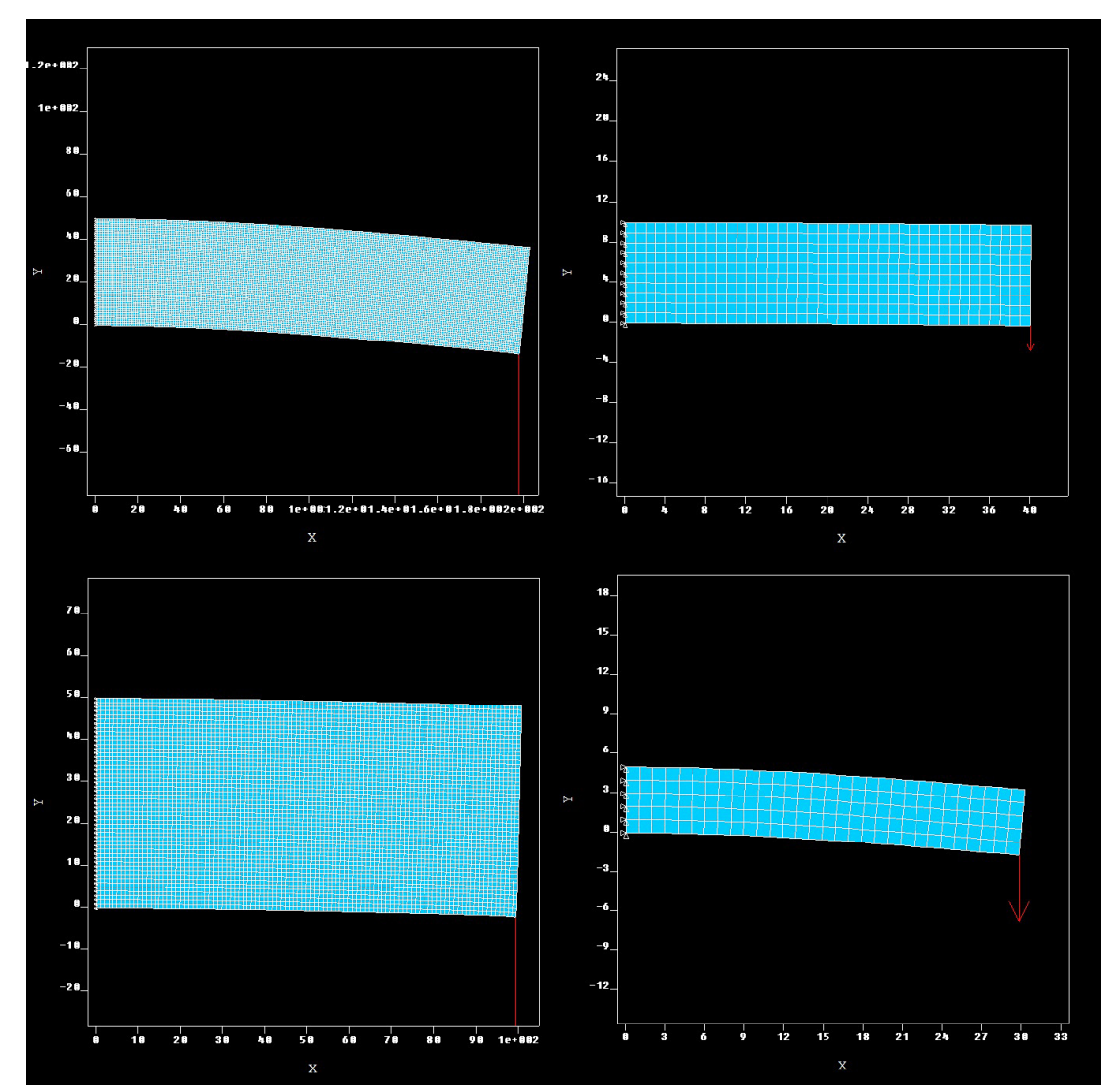

Fig. 1. Possible simple modifications of the beam and force used during project classes 
merical algorithms would not be as complex as in the ANSYS applications. Instead, various basic numerical algorithms well-known to students should be used, e.g. conjugate gradient method, Gauss-Seidel method, Jacobi method or successive over-relaxation method in order to extend students' understanding of numerical methods at the same time. For each of alternative algorithms used, it is possible to analyse the calculations and how some variables change with every iteration of the running program. A sample summary of used methods is shown in Figure 2. This exercise perfectly presents the issue of stability of numerical methods and fixes it in the students' memory. For example, in Figure 3 it is clearly visible that in another analysed case the Jacobi method is not stable, while successive over-relaxation method provides reasonable results.

When students experience the complexity of FEM by writing their own computer programs, they will most likely use ANSYS more knowingly, with the awareness of all the algorithms used in it that are invisible to the user. Again, during any ANSYS analysis it is possible to visualise the problem of discretisation in a more sophisticated tool. It allows the user to modify the grid extensively and precisely to ensure its perfect adjust-

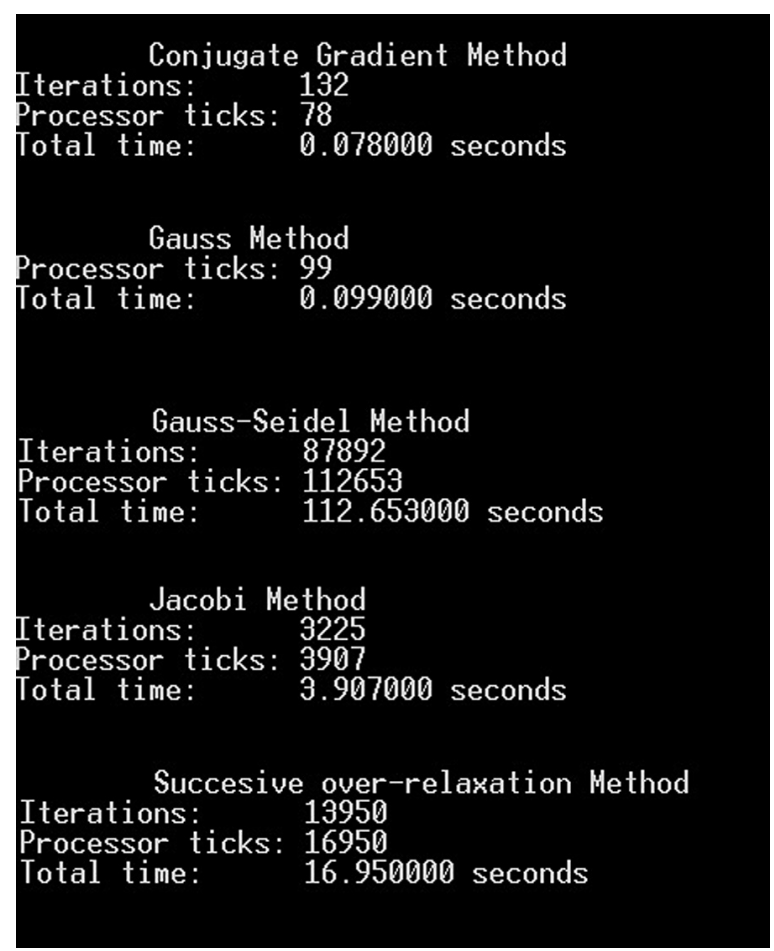

Fig. 2. Example of information that might be gained during project classes with an exemplary code comparison of running times and number of iterations needed for different numerical methods ment to the needs of conducted simulation. What is more, it provides a rich library of available finite elements. Therefore, it is possible to consider just one model using different grids again and again and this way demonstrating how sensitive the finite element method algorithm is to changes of the elements.

\section{SAMPLE ANSYS ANALYSIS - MODELING STRESS AND DEFORMATION OF A LOADED MARS ROVER CHASSIS}

One of the latest innovations of ANSYS worth mentioning is Workbench - a platform designed to integrate various ANSYS applications and this

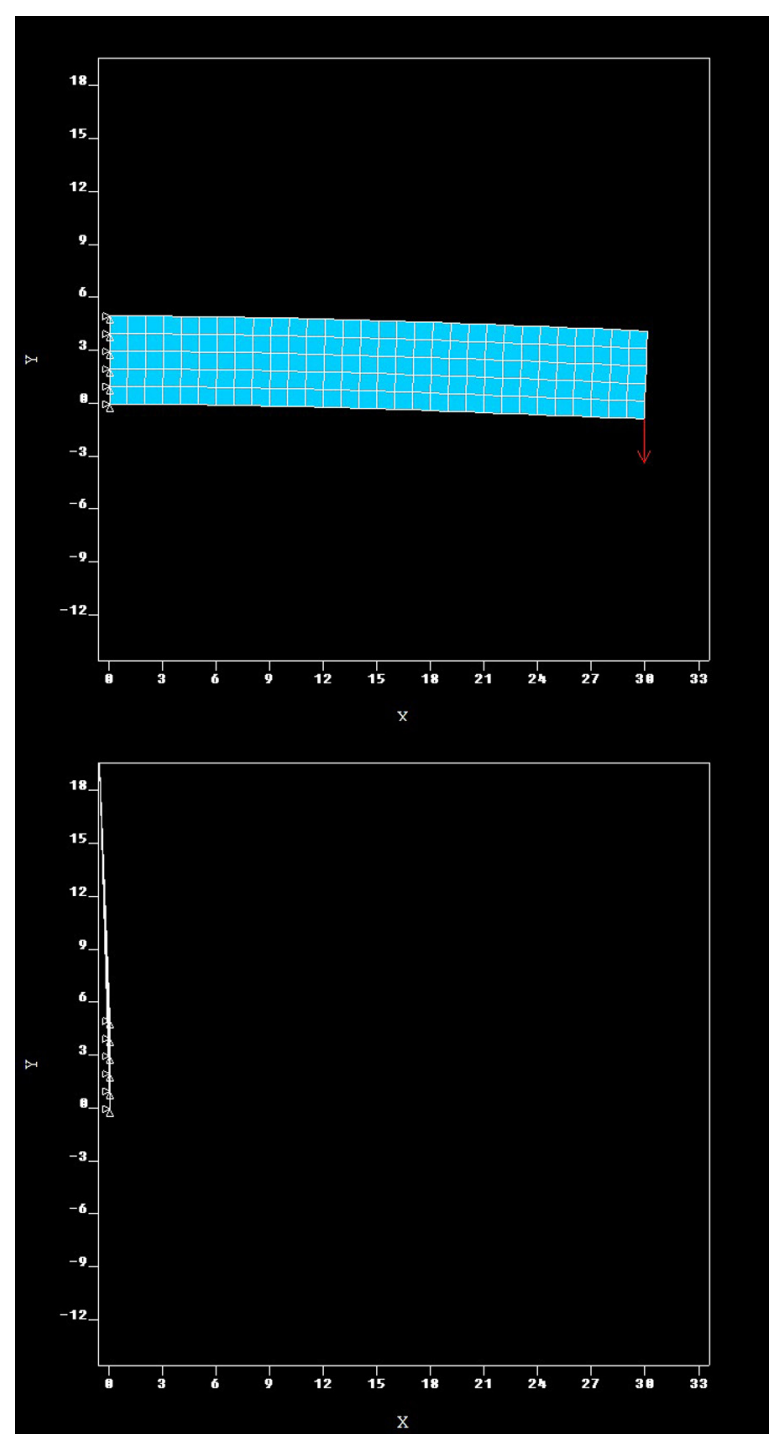

Fig. 3. Comparison of solutions received after using two different numerical methods for identical beams and forces - successive over-relaxation method (above) and Jacobi method (below). The divergence of the Jacobi method is clearly visible 
way increase productivity [5]. The platform provides functionalities of all available products and additionally some basic tools for managing the operations [1]. The platform is exceptionally intuitive thanks to its block diagram visualisation of following steps of the analysis. This chapter presents a sample analysis of a Mars rover's chassis with the use of ANSYS Workbench Mechanical, a product for structural analysis which provides simulations of stress, fatigue and heat transfer. Figures presented below show most important steps of such an analysis - discretisation (meshing) and output solution.
The mesh visible in Figure 4 was condensed in the areas where the chassis shall be integrated with other components of the mobile platform and where notches occur since these are the areas particularly vulnerable to stress concentrations. The analysis contains two different loads. First case is bending the chassis by a force applied on the front wheels. Figure 5 illustrates the equivalent non-Mises stress distribution. The solutions make it possible to predict which areas should be strengthened and where is it possible to place lightening holes or how to modify the design to optimise it, which is often the conclusion of scien-

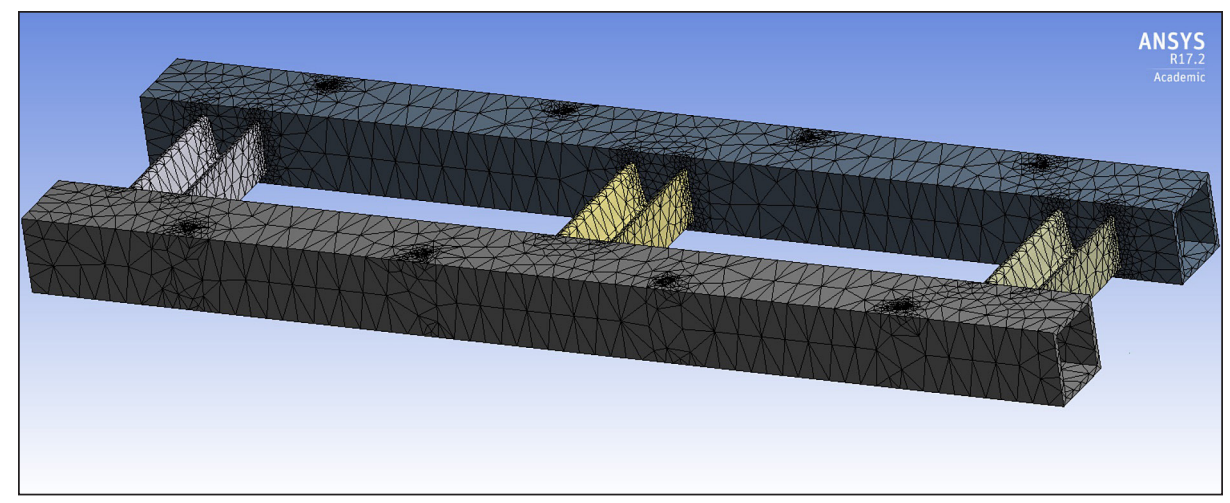

Fig. 4. Example mesh of a chassis of a Mars rover analog condensed in notch areas where stress concentrations are expected

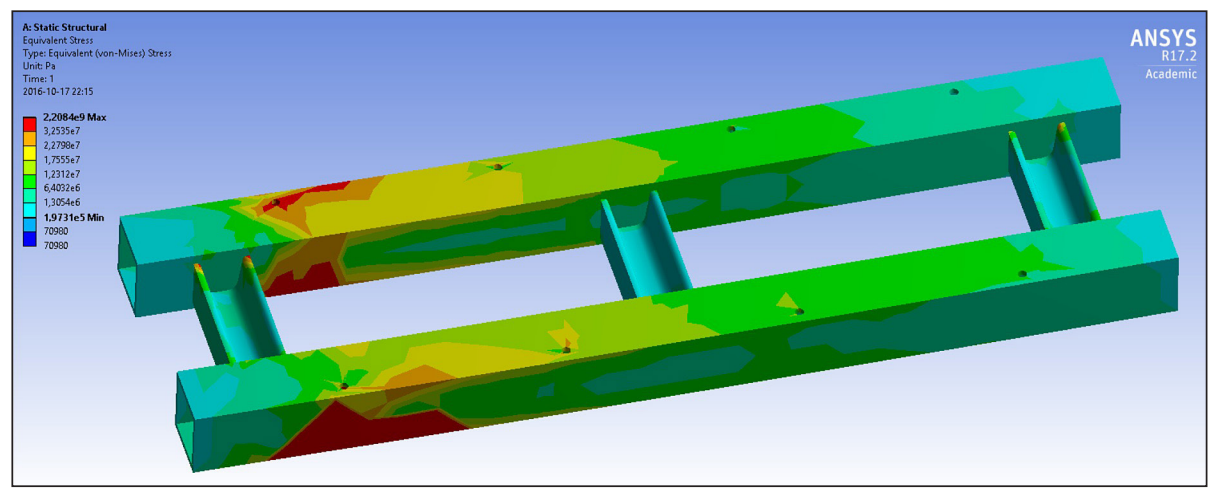

Fig. 5. Example of ANSYS solution - equivalent non-Mises stress for a force applied on front wheels (on the right)

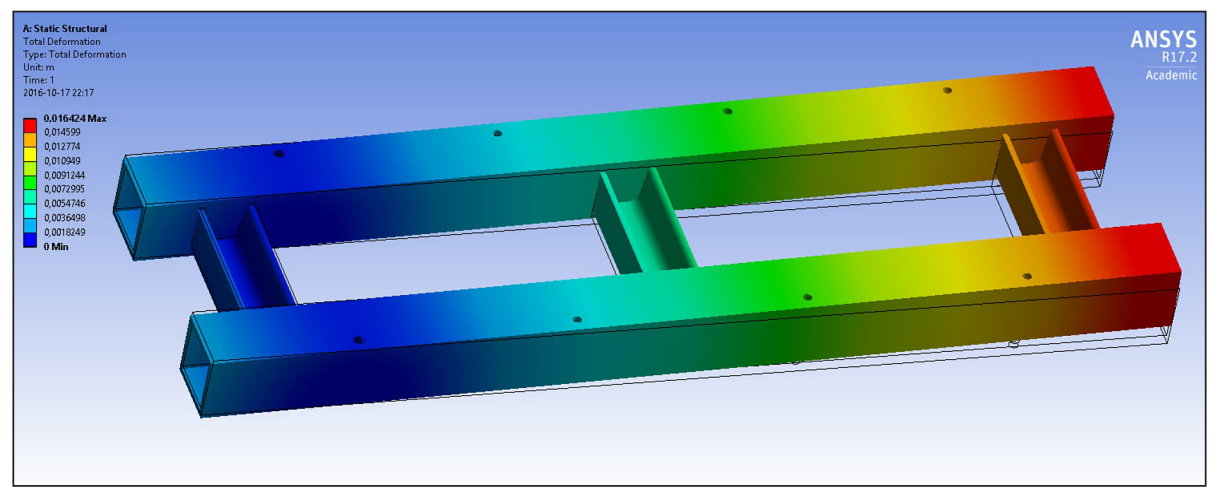

Fig. 6. Example of ANSYS solution - total deformation for a force applied on front wheels (on the right) 


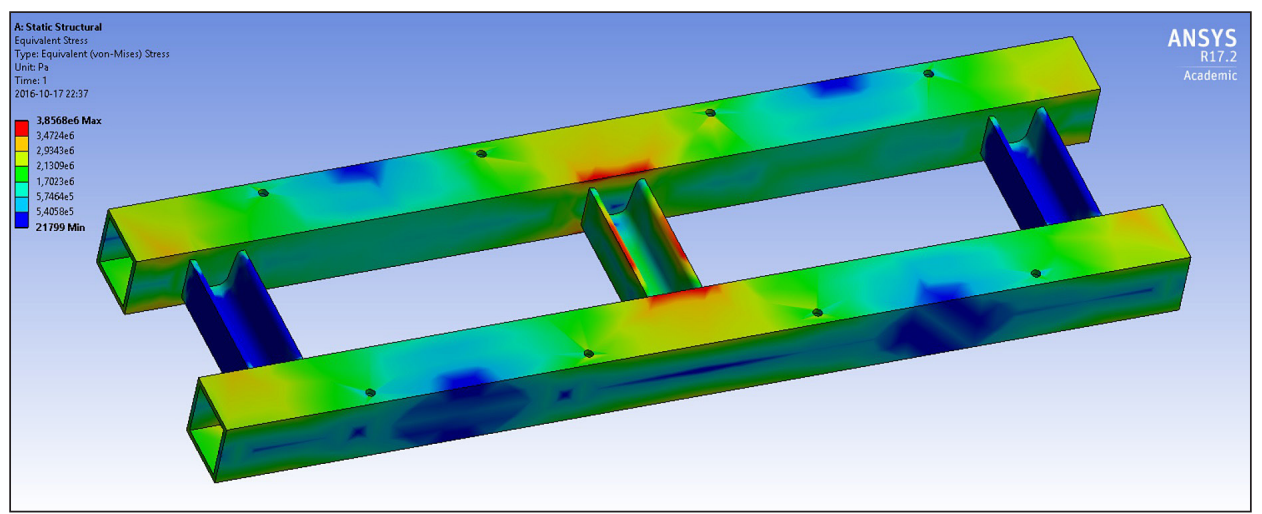

Fig. 7. Example of ANSYS solution - equivalent non-Mises stress for a strong force applied on the centre profile

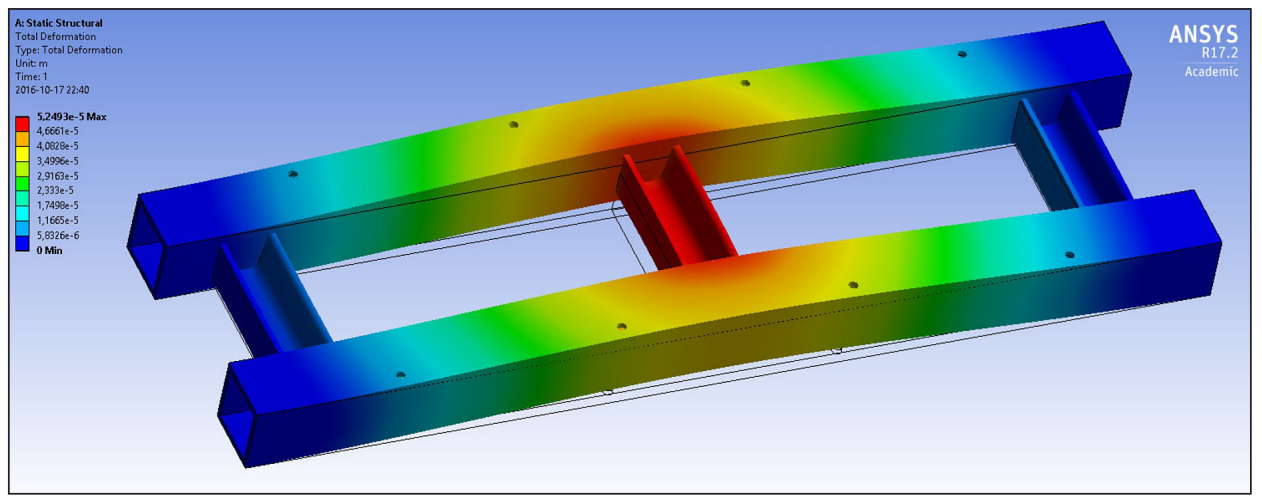

Fig. 8. Example of ANSYS solution - total deformation for a strong force applied on the centre profile

tific papers [7,9]. Figure 6 shows the deformation of the frame in this case. As presented, it is possible to exaggerate the deformed model solution so that the shape of the deformation is clearly visible. The other case analyses bending by a strong force applied on the centre profile of the frame. This situation (although much weaker) may occur when the mobile platform falls from a high setoff and components placed inside the chassis fall down. Figure 7 presents the equivalent non-Mises stress and Figure 8 the exaggerated deformation. Another functionality of ANSYS is checking the exact value of stress in any chosen point of the model in order to determine how close it is to the material's elastic limit. Figures help in concluding that the frame ought not to have any lightening holes cut and that the material used should ensure great strength of the welded joints.

\section{CONCLUSIONS}

Finite element method became a base, thanks to which sophisticated engineering tools like ANSYS could be developed. It is impossible to imagine contemporary engineering without them.
However, it is possible to reverse the order of this sequence and make ANSYS helpful in finite element method. It does not have to stimulate its development and improvement, but is a perfect starting point for presenting the relationships between various algorithm parameters and the results in order to make the issue more understandable to students and extend the number of ANSYS specialists.

Thanks to this software students will be able to analyse any complex problem and receive clear output solutions as presented in the paper. ANSYS analysis is a vital step in every design process in order to optimise the design and ensure safety of the construction.

\section{REFERENCES}

1. ANSYS Products. Available: http://www.ansys. com/Products/All-Products [Accessed: 23.09.2016].

2. Bąk R. and Burczyński T. Strength of materials with elements of computer application (in Polish). WNT, Warszawa 2001.

3. Górecki B. and Łaniewski-Wołłk Ł. Instructions for Numerical Methods laboratories (in Polish). Division of Aerodynamics, Faculty of Power and 
Aeronautical Engineering, Warsaw University of Technology. Available online: https://www.meil. pw.edu.pl/pl/ZA [Accessed: 15.05.2016].

4. Kleiber M. Intruduction to the Finite Element Method (in Polish). IPPT PAN, PWN, Warszawa 1989.

5. Krzesiński G., Zagrajek T., Marek P. and Borkowski P. Finite Element Method in mechanics of materials and structural mechanics. Solving sample problems with the use of ANSYS (in Polish). OWPW, 2015.

6. Rakowski G. and Kacprzyk Z. Finite Element Method in structural mechanics (in Polish).
OWPW, 1993.

7. Różyło P. and Wrzesińska K. Numerical analysis of the behavior of compressed thin-walled elements with holes. Advances in Science and Technology Research Journal, 10(31), 2016, 199-206.

8. Rusiński E., Czmochowski J. and Smolnicki T. Advanced Finite Element Method in supporting structures (in Polish). Oficyna Wydawnicza Politechniki Wrocławskiej, 1999.

9. Ziobro J. Analysis of suspension element of car body on the example silentblock. Advances in Science and Technology Research Journal, 9(28), 2015, 125-129. 